\title{
Supporting Information for Adsorption of Cadmium Using Biochars Produced From Agro-Residues
}

\author{
Alejandra Rendón-Calle, ${ }^{\mathrm{a}, \mathrm{b}}$ Santiago Builes, ${ }^{\mathrm{a} \dagger}$ and Federico Calle-Vallejo ${ }^{\mathrm{b} \dagger}$ \\ Julián E. López ${ }^{1}$, Santiago Builes ${ }^{2 \dagger}$, Mario A. Heredia Salgado ${ }^{3}$, Luís A.C Tarelho ${ }^{3}$, \\ Catalina Arroyave ${ }^{1}$, Adriana Aristizábal ${ }^{2}$, Eduardo Chavez $^{4}$ \\ ${ }^{1}$ Environmental Engineering Faculty, Universidad de Medellín, Medellín, 050026, Colombia \\ 2 Departamento de Ingeniería de Procesos, Universidad EAFIT, Carrera 49 No 7 sur-50, 050022, \\ Medellín, Colombia. \\ ${ }^{3}$ Department of Environment and Planning, Centre for Environmental and Marine Studies (CESAM), \\ University of Aveiro, 3810-193, Portugal \\ ${ }^{4}$ Escuela Superior Politécnica del Litoral, ESPOL, Facultad de Ciencias de la Vida, Campus Gustavo \\ Galindo Km. 30.5 Vía Perimetral, P.O. Box 09-01-5863, Guayaquil, Ecuador. \\ $\dagger$ †mails: sbuiles@eafit.edu.co
}

Table of contents

page

$\begin{array}{ll}\text { S1. Quality assurance and quality control } & \text { S2 }\end{array}$

S2. SEM and EDS characterization $\quad$ S2

S3. XRD analysis $\quad$ S4

S4. FTIR analysis $\quad$ S5

References $\quad$ S6 


\section{S1. Quality assurance and quality control}

To assure the reliability of the results, quality assurance and quality control protocols were included throughout the analytical processes. All reagents were of analytical. Four certified synthetic solutions (CSS) for trace elements (Sigma-Aldrich $®$ ) were used for ICP-OES calibration. Calibration curve was accepted at R2 $>0.9996$ (for Cd). CSS and samples duplicates were included every 20 samples for quality assurance and evaluate reproducibility, respectively. The glassware was soaked in nitric acid $\left(3 \% \mathrm{HNO}_{3}\right)$ bath overnight prior use.

\section{S2. SEM and EDS characterization}

The post-sorption biochar samples were used to identify by SEM/EDS the phases present in the biochar. Figure S1 shows the SEM images analyzed and the corresponding EDS spectra. 

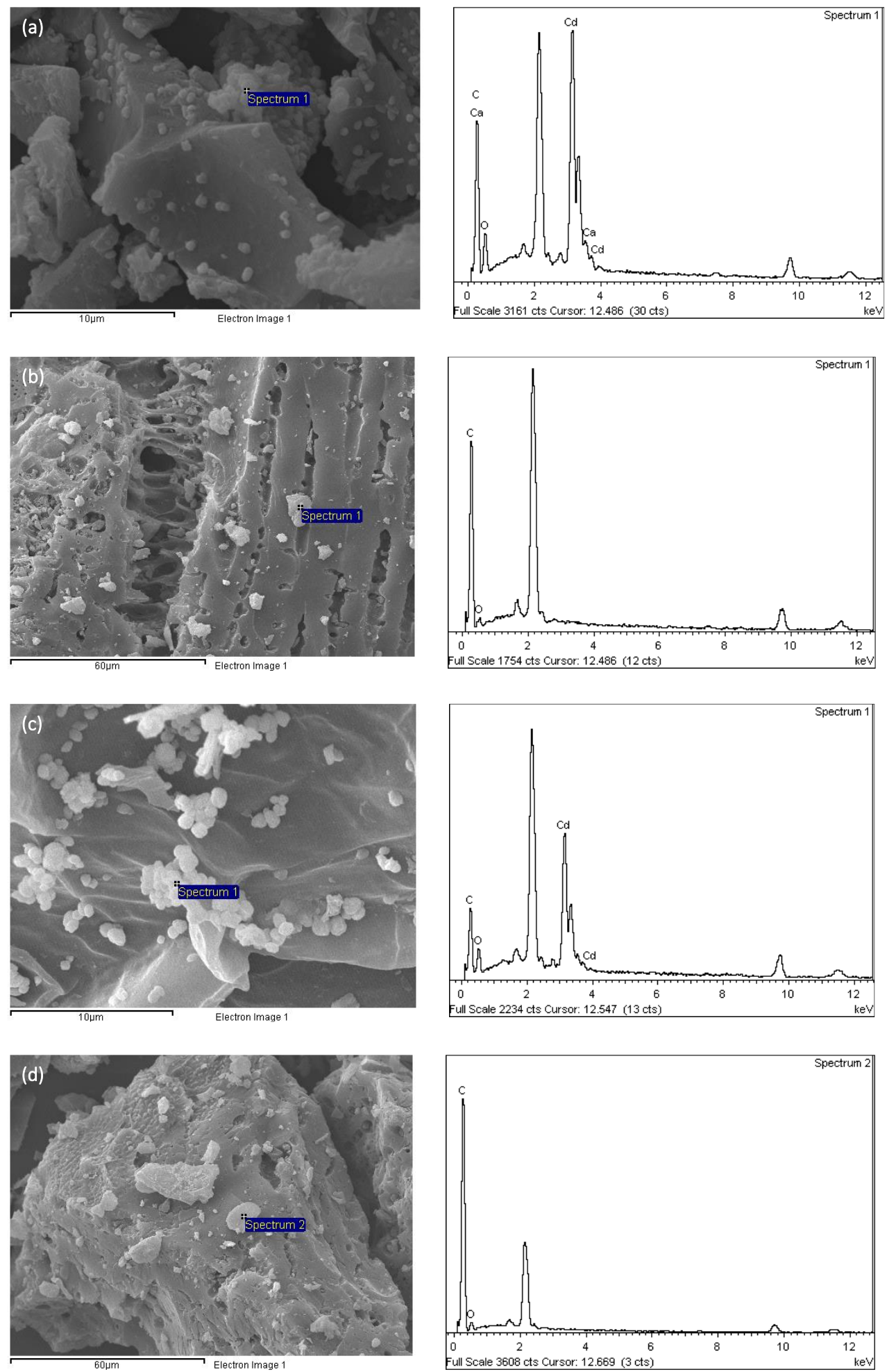

Figure S1. SEM image (left) and corresponding EDS spectra (right) of post-sorbed materials (with a cadmium equilibrium concentration in the solution of around $50 \mathrm{mg} / \mathrm{l}$ ) 
for $\mathrm{CH}(\mathrm{a}), \mathrm{PKS}+\mathrm{C}$ (b), QN (c) and PKS (d). The SEM images shows the location for the EDS spectra depicted.

\section{S3. XRD analysis}

The crystals identified in the previous section were analyzed by XRD to identify the crystalline phases present in the biochars as shown in Figure S2. For PKS and PKS+C biochars it was not possible to carry out crystalline phase identification, due to the amorphous nature of the materials.

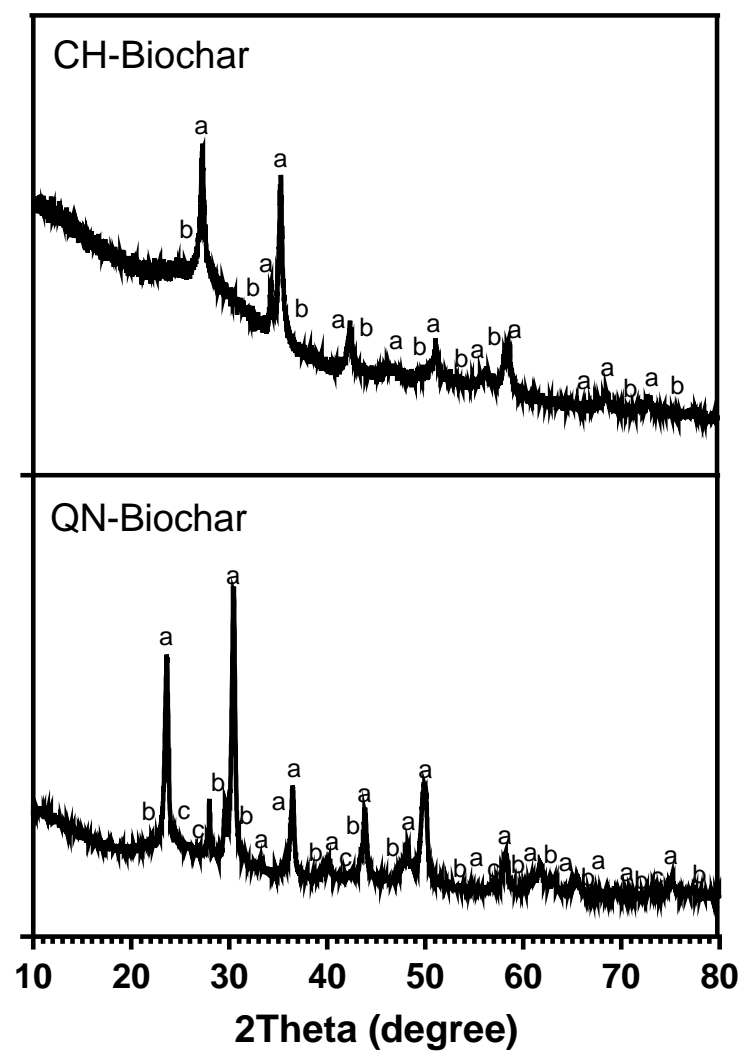

Figure S2. X-ray diffraction patterns of post-sorbed $\mathrm{CH}$ and $\mathrm{QN}$ biochars (with a cadmium equilibrium concentration in the solution of around $50 \mathrm{mg} / \mathrm{l})$. The crystalline phases with typical peaks as: Otavite (a), Calcite (b), Sylvine (c). 


\section{S4. FTIR analysis}

After cadmium sorption, a decrease in intensity of the bands at 1030, 1600 and 2900 $\mathrm{cm}^{-1}$ in $\mathrm{CH}$ and QN biochars was observed (See Figure S3). This, suggest that oxygencontaining functional groups are involved in the cadmium sorption process. The band at $1600 \mathrm{~cm}^{-1}$ shifted to $1590 \mathrm{~cm}^{-1}$ in the PKS+C and PKS biochars and the intensity of the peak at $1030 \mathrm{~cm}^{-1}$ decreased. This is in agreement with the results of Cui et al. [1] The authors found that after cadmium sorption, a sharp shift occurred to the peaks of aromatic functional groups $\left(\mathrm{C}-\mathrm{H}, \mathrm{CH}_{2}\right.$ and $\left.\mathrm{C}=\mathrm{C}\right)$. These results highlight the importance of the $\mathrm{Cd}^{2+}-\pi$ interaction on the cadmium sorption process.

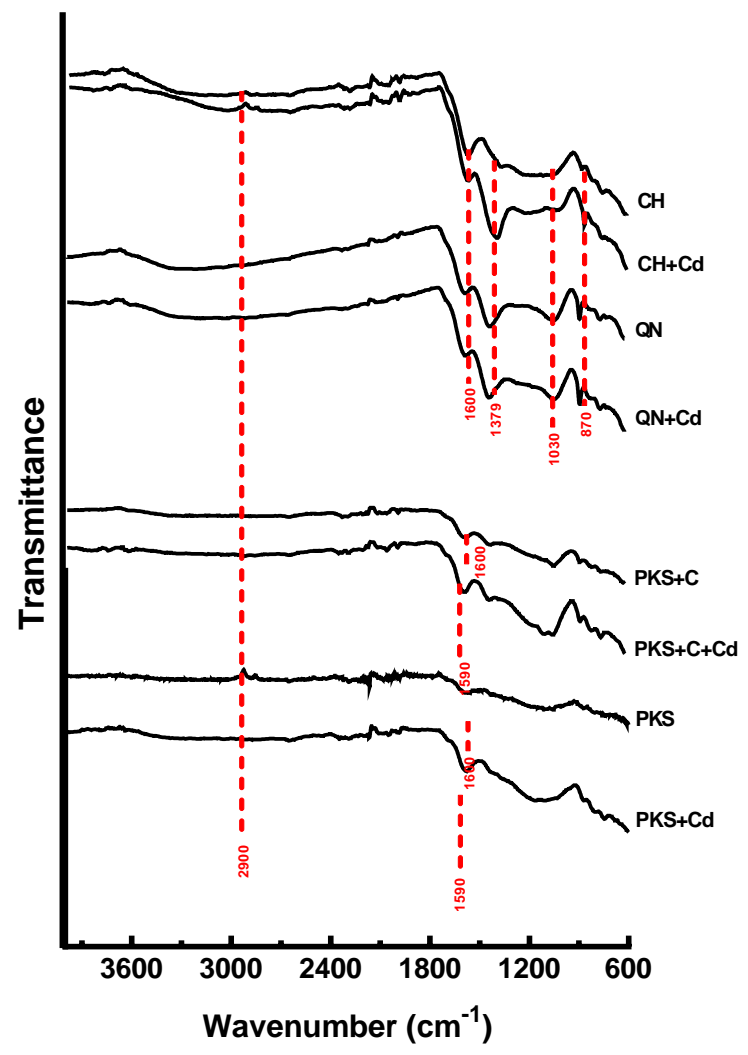

Figure S3. FTIR spectra of biochar samples QN, CH, PKS and PKS $+\mathrm{C}$ before (top) and after (bottom) cadmium sorption 


\section{References}

[1] X. Cui, S. Fang, Y. Yao, T. Li, Q. Ni, X. Yang, et al., "Potential mechanisms of cadmium removal from aqueous solution by Canna indica derived biochar," Science of The Total Environment, vol. 562, pp. 517-525, 2016/08/15/ 2016. 